\title{
On Reliability of $(n+1)$-unit Warm Standby System based on Imperfect Repair Facility and Two Types of Failures
}

\author{
M. A. W. Mahmoud \\ Mathematics Department, \\ Faculty of Science, Al-Azhar University, \\ Nasr City (11884), Cairo, Egypt.
}

\author{
M. A. Attallah \\ Department of Statistics, \\ Faculty of Science, \\ King Abdul Aziz University, Jeddah, SA.
}

\author{
A. Alharby \\ Department of Statistics, \\ Faculty of Science, \\ King Abdul Aziz University, Jeddah, SA.
}

\begin{abstract}
This paper deals with an $(n+1)$-unit warm standby system based on imperfect repair facility and two types of failures. These types of failure are hardware and human error failures. Various measures of the system reliability are obtained using the regenerative point technique. Finally a numerical example is presented to illustrate the theoretical results.
\end{abstract}

\section{General Terms:}

Reliability and Economic Measures

\section{Keywords:}

reliability, warmstandby, humanerror failure, hardware failure, availability, cost benefit.

\section{INTRODUCTION}

The theory of reliability is one of the most important branches of operations research and system engineering. Any system is analyzed in order to be complete, must give due consideration to system reliability. With remarkable advances made in electronics engineering, military and communication systems have become more sophisticated and when such systems fail, very serious situations aries. Thus in the present day context, high system reliability has become very important from the viewpoint of both makers and users.

A system designer is faced often with problems of determining the various system measures like reliability, availability . . etc. $\mathrm{He}$ also has to suggest ways that improve the efficiency of a given system. Due to the nature of the subject, the methods of probability theory and mathematical statistics are necessary to study and solve the problems that arise in reliability theory.

Many papers [1, 9, 12, 14, 18] studied the reliability of the twounit standby system from various points of view. In all these papers, the authors did not take into their account the human error failures in spite of $20-30 \%$ of failures are due to human error (see Mister [17]).

In addition, many papers [2] $[8],[13],[15,16]$ dealt with the reliability of some standby systems are subject to the human error failures.
As we know, there is no papers in the literature deal with $(n+1)$ unit standby system in which $n$ units are initially in operation and the other unit is kept as a standby unit. Thus the aim of this paper is to bridge a gab of analyzing $(n+1)$-unit warm standby system based on imperfect repair facility and two types of failures. Various measures of the system reliability such as mean time to system failure, steady-state availability, steady-state busy period and cost benefit analysis, are derived based on regenerative point technique.

Finally, numerical example is given to illustrate the theoretical results.

\section{ASSUMPTIONS}

The following assumptions are associated with the proposed model.

1- The system consists of $n+1$ identical units.

2- Initially $n$ units begin operation and the other unit is kept as warm standby.

3- The switch is perfect and instantaneous. (i.e. it is not fail and the time spend to put the standby in operating is negligible).

4- The operative units suffer two types of failures namely, hardware and human error failures, while standby unit suffers only one type of failure.

5- There is one repair facility one serverman which it is available with probability $P$.

6- After repair the unit is as good as new.

7- All random variables are independent and exponentially distributed

8- The system fails when there are $(n-1)$ operating only.

\section{NOTATIONS}

$\lambda_{i}, i=1,2$ : failure rates of hardware and human error respectively

$\nu_{i}, i=1,2$ : repair rates of repair for hardware and human error failures respectively

$\gamma:$ failure rate of the standby unit

$\eta$ : repair rate of the standby unit

$1-\exp \left(\lambda_{i} t\right), i=1,2: \mathrm{CDF}$ of hardware and human error failures respectively 
$1-\exp \left(-\nu_{i} t\right), i=1,2: \mathrm{CDF}$ of repair due to hardware and human error respectively

$1-\exp (-\gamma t): \mathrm{CDF}$ of standby failure time

$1-\exp (-\eta t): \mathrm{CDF}$ of repair time of the standby unit

$1-\exp (-\xi t): \mathrm{CDF}$ of waiting time for repair facility to be active

$E_{0}:$ state of the system at $t=0$

$E$ : set of regenerative states

$\bar{E}:$ set of non-regenerative states

$P:$ probability that facility the repair is available

$q_{i, j}(t)$ : PDF of time for the system transition from regenerative state $S_{i}$ to $S_{j}$

$Q_{i j}(t): \mathrm{CDF}$ of time for the system transition from regenerative state $S_{i}$ to $S_{j}$

$q_{i j}^{(k)}(t)$ : PDF of time for the system transition from regenerative state $S_{i}$ to $S_{j}$ via state $S_{k}$

$q_{i j}^{(k, I)}(t)$ : PDF of time for the system transition from regenerative state $S_{i}$ to $S_{j}$ via two non-regenerative state $S_{k}$ and $S_{I}$

$\mu_{i}: \int P$ (System sojourns in states $S_{i}$ of the set $E$ for at least time $t) d t$

$M_{i}(t): P$ (System is up initially in state $S_{i}$ of the set $E$ is up at time $T$ without passing through any other regenerative state or returning to itself through on or more states of $E$ )

$A V_{i}(t): P$ (system is up to time $t \mid E_{0}=S_{i}$ of $E$ )

$B_{i}^{1}(t): P$ (the serverman is busy with repair due to hardware failure)

$B_{i}^{2}(t): P$ (the serverman is busy with repair due to haman error failure)

$B_{i}^{3}(t): P$ (the serverman is busy with standby repair)

$\Pi_{i}(t)$ : CDF of time to system failure starting from state $S_{i}$

$u$ : dummy variable in Laplase transform $(L T)$

* : symbol for $L T$

(C) : symbol for convolution

St $:$ unit is in standby case

$r_{i},(i=1,2):$ unit is under repair due to hardware failure and human error failure respectivey

$R$ : repair continued from earlier state

$S r:$ the standby unit is under repair

$S R$ : the repair of standby unit continued from earlier state

$W r$ : the unit is waiting for repair

$S W r$ : the standby unit is waiting for repair

$r f g$ : the repair facility is available

$r f b$ : the repair facility is not available

$W R$ : the unit is waiting for repair from earlier state

$S W R$ : the standby unit is waiting for repair from earlier state

$O_{n}: n$ units are operating.

The proposed system can be in one of the following states: $S_{0} \equiv\left(O_{n}, S t\right), S_{1} \equiv\left(O_{n}, r_{1}, r f g\right), S_{2} \equiv\left(O_{n}, r_{2}, r f g\right)$, $S_{3} \equiv\left(O_{n}, S r, r f g\right), S_{4} \equiv\left(O_{0}, W r_{1}, r f b\right)$,

$S_{5} \equiv\left(O_{n}, W r_{2}, r f b\right), \quad S_{6} \equiv\left(O_{n}, S W r, r f b\right)$

$S_{7} \equiv\left(O_{n-1}, R_{1}, w r_{1}\right), \quad S_{8} \equiv\left(O_{n-1}, R_{1}, w r_{2}\right)$,

$S_{9} \equiv\left(O_{n-1}, R_{2}, w r_{1}\right), S_{10} \equiv\left(O_{n-1}, R_{2}, w r_{2}\right)$,

$S_{11} \equiv\left(O_{n-1}, W R_{1}, w r_{1}\right), S_{12} \equiv\left(O_{n-1}, W R_{1}, w r_{2}\right)$,

$S_{13} \equiv\left(O_{n-1}, W R_{2}, w r_{1}\right), S_{14} \equiv\left(O_{n-1}, W R_{2}, w r_{2}\right)$,

$S_{15} \equiv\left(O_{n-1}, S R, w r_{1}\right), S_{16} \equiv\left(O_{n-1}, S R, w r_{2}\right)$,

$S_{17} \equiv\left(O_{n-1}, S W R, w r_{1}\right), S_{18} \equiv\left(O_{n-1}, S W R, w r_{2}\right)$

REMARK 1. 1. The states $S_{0}-S_{6}$ are regenerative states.

2. The states $S_{7}-S_{18}$ are non-regenerative states.

\section{THE TRANSITION PROBABILITIES}

It can be observed that the points of entry into any of the states $S_{i}$ of the set $E$ are regenerative points. Let $T_{0}(\equiv 0), T_{1}, T_{2}, \cdots$ denote the epochs at which the system enters any state $S_{i}$ of $E$ and let $X_{n}$ denote the state visited at epoch $T_{n}+$ (i.e. just after transition at $T_{n}$ ). It is easy to see that $\left\{X_{n}, T_{n}\right\}$ is a Markov renewal process with state space $E$ and

$$
Q_{i j}(t)=P\left[X_{n+1}=j, T_{n+1}-T_{n} \leq t \mid X_{n}=t\right],
$$

is the semi Markov kernel over $E$.

The matrix of transition probabilities $\mathrm{s}$ given by

$$
P=\left(P_{i j}\right)=\left(Q_{i j}(\infty)\right)=Q(\infty),
$$

with nonzero elements. It is easy to obtain

$$
P_{01}=\frac{n p \lambda_{1}}{n \lambda_{1}+n \lambda_{2}+\gamma} .
$$

Similarly

$$
\begin{array}{ll}
P_{02}=\frac{n p \lambda_{2}}{n \lambda_{1}+n \lambda_{2}+\gamma}, P_{03}=\frac{p \gamma}{n \lambda_{1}+n \lambda_{2}+\gamma}, P_{04}=\frac{n q \lambda_{1}}{n \lambda_{1}+n \lambda_{2}+\gamma}, \\
P_{05}=\frac{n \lambda_{2}}{n \lambda_{1}+n \lambda_{2}+\gamma}, P_{06}=\frac{q \lambda_{1}}{n \lambda_{1}+n \lambda_{2}+\gamma}, P_{17}=\frac{n \lambda_{1}+n \lambda_{2}+\gamma_{1}}{n \lambda_{1}} . \\
P_{18}=\frac{n \lambda_{2}}{n \lambda_{1}+n \lambda_{2}+\gamma_{1}}, & P_{10}=\frac{\gamma_{1}}{n \lambda_{1}+n \lambda_{2}+\gamma_{1}}, \\
P_{11}^{(7)}=\frac{n \lambda_{1}}{n \lambda_{1}+n \lambda_{2}+\gamma_{1}}=P_{17}, & P_{12}^{(8)}=\frac{n \lambda_{2}}{n \lambda_{1}+n \lambda_{2}+\gamma_{1}}=P_{18}, \\
P_{20}=\frac{\gamma_{2}}{n \lambda_{1}+n \lambda_{2}+\gamma_{2}}, & P_{29}=\frac{n \lambda_{1}}{n \lambda_{1}+n \lambda_{2}+\gamma_{2}}, \\
P_{2,10}=\frac{n \lambda_{2}}{n \lambda_{1}+n \lambda_{2}+\gamma_{2}}, & P_{21}^{(9)}=\frac{n \lambda_{1}}{n \lambda_{1}+n \lambda_{2}+\gamma_{2}}=P_{29}, \\
P_{22}^{(10)}=\frac{n \lambda_{2}}{n \lambda_{1}+n \lambda_{2}+\gamma_{2}}=P_{2,10}, & P_{3,15}=\frac{n \lambda_{1}}{n \lambda_{1}+n \lambda_{2}+\eta}, \\
P_{3,16}=\frac{n \lambda_{2}}{n \lambda_{1}+n \lambda_{2}+\eta}, & P_{3,0}=\frac{\eta}{n \lambda_{1}+n \lambda_{2}+\eta}, \\
P_{31}^{(15)}=\frac{n \lambda_{1}}{n \lambda_{1}+n \lambda_{2}+\eta}=P_{3,15}, & P_{32}^{(16)}=\frac{n \lambda_{2}}{n \lambda_{1}+n \lambda_{2}+\eta}=P_{3,16}, \\
P_{41}=\frac{\xi}{n \lambda_{1}+n \lambda_{2}+\xi}, & P_{4,11}=\frac{n \lambda_{1}}{n \lambda_{1}+n \lambda_{2}+\xi}, \\
P_{4,12}=\frac{n \lambda_{2}}{n \lambda_{1}+n \lambda_{2}+\xi}, & P_{41}^{(11,7)}=\frac{n \lambda_{1}}{n \lambda_{1}+n \lambda_{2}+\xi}=P_{4,11}, \\
P_{42}^{(12,8)}=\frac{n \lambda_{2}}{n \lambda_{1}+n \lambda_{2}+\xi}=P_{4,12}, & P_{52}=\frac{\xi}{n \lambda_{1}+n \lambda_{2}+\xi}=P_{41}, \\
P_{5,13}=P_{4,11}, & P_{5,14}=P_{4,12}, \\
P_{51}^{(13,9)}=P_{42}^{(12,8)}, & P_{6,3}=P_{4,1}, \\
P_{6,17}=P_{4,11}, & P_{6,18}=P_{4,12}, \\
P_{61}^{(17,15)}=P_{6,17}=P_{4,11}, & P_{62}^{(18,16)}=P_{6,18}=P_{4,12} .
\end{array}
$$

\section{THE MEAN SOJOURN TIMES}

One can show that

$$
\begin{aligned}
\mu_{0} & =\frac{1}{n \lambda_{1}+n \lambda_{2}+\gamma}, \\
\mu_{1} & =\frac{1}{n \lambda_{1}+n \lambda_{2}+\mu_{1}}, \\
\mu_{2} & =\frac{1}{n \lambda_{1}+n \lambda_{2}+\mu_{2}}, \\
\mu_{3} & =\frac{1}{n \lambda_{1}+n \lambda_{2}+\eta}, \\
\mu_{4}=\mu_{5} & =\mu_{6}=\frac{1}{n \lambda_{1}+n \lambda_{2}+\xi} .
\end{aligned}
$$

\section{THE MEAN TIME TO SYSTEM FAILURE}

In this section, we derive the mean time to failure MTTF. Upon using the probability theory we get

$$
\begin{gathered}
\bar{\Pi}_{0}(t)=e^{-\left(n \lambda_{1}+n \lambda_{2}+\gamma\right) t}+\sum_{i=1}^{6} q_{0 i}(t)\left(\bar{\Pi}_{1}(t),\right. \\
\bar{\Pi}_{1}(t)=e^{-\left(n \lambda_{1}+n \lambda_{2}+\nu_{1}\right) t}+q_{10}(t)\left(\bar{\Pi}_{0}(t),\right. \\
\bar{\Pi}_{2}(t)=e^{-\left(n \lambda_{1}+n \lambda_{2}+\nu_{2}\right) t}+q_{20}(t)\left(\mathcal{C} \bar{\Pi}_{0}(t),\right.
\end{gathered}
$$




$$
\begin{aligned}
& \bar{\Pi}_{3}(t)=e^{-\left(n \lambda_{1}+n \lambda_{2}+\eta\right) t}+q_{30}(t) @ \bar{\Pi}_{0}(t), \\
& \bar{\Pi}_{4}(t)=e^{-\left(n \lambda_{1}+n \lambda_{2}+\xi\right) t}+q_{41}(t) @ \bar{\Pi}_{1}(t), \\
& \bar{\Pi}_{5}(t)=e^{-\left(n \lambda_{1}+n \lambda_{2}+\xi\right) t}+q_{52}(t) @ \bar{\Pi}_{2}(t), \\
& \bar{\Pi}_{6}(t)=e^{-\left(n \lambda_{1}+n \lambda_{2}+\xi\right) t}+q_{63}(t) @ \bar{\Pi}_{3}(t) .
\end{aligned}
$$

Taking Laplace transform of both sides of the system (6)-(12), setting $u=0$ and solving for $\bar{\Pi}_{0}^{*}(0)$ yields

$$
\begin{gathered}
\bar{\Pi}_{0}^{*}(0)=M T T F=\frac{L_{2}}{L_{1}}, \\
L_{1}=1-P_{01} P_{10}-P_{02} P_{20}-P_{03} P_{30}-P_{04} P_{41} P_{10} \\
-P_{05} P_{52} P_{20}-P_{06} P_{63} P_{30}, \\
L_{2}=\mu_{0}+a_{1} \mu_{1}+a_{2} \mu_{2}+a_{3} \mu_{3}+P_{04} \mu_{4}+P_{05} \mu_{5}+P_{06} \mu_{6}, \\
\text { where } \\
a_{1}=P_{01}+P_{04} P_{41}, a_{2}=P_{02}+P_{05} P_{52}, a_{3}=P_{03}+P_{06} P_{63} .
\end{gathered}
$$

\section{SYSTEM AVAILABILITY}

Using probabilistic arguments, gives

$$
A V_{0}(t)=M_{0}(t)+\sum_{i=1}^{6} q_{0 i}(t)\left(A V_{i}(t),\right.
$$

$$
\begin{aligned}
A V_{1}(t)= & M_{1}(t)+q_{10}(t) A V_{0}(t)+q_{11}^{(7)}(t) A V_{1}(t) \\
& +q_{12}^{(8)}(t) A V_{2}(t),
\end{aligned}
$$

$$
\begin{aligned}
A V_{2}(t)= & M_{2}(t)+q_{20}(t) A V_{0}(t)+q_{21}^{(9)}(t) A V_{1}(t) \\
& +q_{22}^{(10)}(t) A V_{2}(t),
\end{aligned}
$$

$$
\begin{aligned}
A V_{3}(t)= & M_{3}(t)+q_{30}(t) A V_{0}(t)+q_{31}^{(15)}(t) A V_{1}(t) \\
& +q_{32}^{(16)}(t) A V_{2}(t),
\end{aligned}
$$

$$
\begin{aligned}
A V_{4}(t)= & M_{4}(t)+q_{41}(t) A V_{1}(t)+q_{41}^{(11,7)}(t) A V_{1}(t) \\
& +q_{42}^{(12,8)}(t) A V_{2}(t),
\end{aligned}
$$$$
A V_{5}(t)=M_{5}(t)+q_{52}(t) A V_{2}(t)+q_{51}^{(13,9)}(t) A V_{1}(t)
$$$$
+q_{52}^{(14,10)}(t) A V_{2}(t)
$$

$$
\begin{aligned}
A V_{6}(t)= & M_{6}(t)+q_{63}(t) A V_{3}(t)+q_{61}^{(17,15)}(t) A V_{1}(t) \\
& +q_{62}^{(18,16)}(t) A V_{2}(t),
\end{aligned}
$$

where

$$
\begin{gathered}
M_{0}(t)=\exp \left[-\left(n \lambda_{1}+n \lambda_{2}+\gamma\right) t\right], \\
M_{1}(t)=\exp \left[-\left(n \lambda_{1}+n \lambda_{2}+\mu_{1}\right) t\right], \\
M_{2}(t)=\exp \left[-\left(n \lambda_{1}+n \lambda_{2}+\mu_{2}\right) t\right], \\
M_{3}(t)=\exp \left[-\left(n \lambda_{1}+n \lambda_{2}+\eta\right) t\right], \\
M_{4}(t)=\exp \left[-\left(n \lambda_{1}+n \lambda_{2}+\xi\right) t\right]=M_{5}(t)=M_{6}(t) .
\end{gathered}
$$

Taking Laplace transform of both sides of Eqs. (14)-(20) and solving for $A V_{0}^{*}(u)$, the steady-state availability of the system $A V_{0}$ can be given by

$$
A V_{0}=\lim _{t \rightarrow \infty} A V_{0}(t)=\lim _{u \rightarrow 0} u A V_{0}^{*}(u)=\frac{N_{0}}{D_{0}},
$$

where

$$
\begin{aligned}
& N_{0}=\mu_{0}+\mu_{1} P_{01}+\mu_{2} P_{02}+\mu_{3} P_{03}+\mu_{4} P_{04}+\mu_{5} P_{05}+\mu_{6} P_{06} \\
& -\mu_{0} P_{17}-\mu_{2} P_{02} P_{17}-\mu_{3} P_{03} P_{17}-\mu_{4} P_{04} P_{17}-\mu_{5} P_{05} P_{17} \\
& -\mu_{6} P_{06} P_{17}+\mu_{2} P_{01} P_{18}-\mu_{0} P_{2,10}-m u_{1} P_{01} P_{2,10} \\
& -\mu_{3} P_{03} P_{2,10}-\mu_{4} P_{04} P_{2,10}-\mu_{5} P_{05} P_{2,10}-\mu_{6} P_{06} P_{2,10} \\
& +\mu_{0} P_{17} P_{2,10}+\mu_{3} P_{03} P_{17} P_{2,10}+\mu_{4} P_{04} P_{17} P_{2,10} \\
& +\mu_{5} P_{05} P_{17} P_{2,10}+\mu_{6} P_{06} P_{17} P_{2,10}+\mu_{1} P_{02} P_{29} \\
& -\mu_{0} P_{18} P_{29}-\mu_{3} P_{03} P_{18} P_{29}-\mu_{4} P_{04} P_{18} P_{29} \\
& +\mu_{5} P_{05} P_{18} P_{29}+\mu_{6} P_{06} P_{18} P_{29}+\mu_{1} P_{03} P_{3,15} \\
& +\mu_{2} P_{03} P_{3,16}-\mu_{2} P_{03} P_{17} P_{3,16}+\mu_{1} P_{03} P_{29} P_{3,16} \\
& +\mu_{1} P_{04} P_{41}+\mu_{2} P_{04} P_{18} P_{41}-\mu_{1} P_{04} P_{2,10} P_{41} \\
& -\mu_{1} P_{04} P_{4,11}+\mu_{2} P_{04} P_{18} P_{4,11}-\mu_{1} P_{04} P_{2,10} P_{4,11} \\
& +\mu_{2} P_{04} P_{4,12}-\mu_{2} P_{04} P_{17} P_{4,12}+\mu_{1} P_{04} P_{29} P_{4,12} \\
& +\mu_{1} P_{05} P_{5,13}+\mu_{2} P_{05} P_{18} P_{5,13}-\mu_{1} P_{05} P_{2,10} P_{5,13} \\
& +\mu_{2} P_{05} P_{5,14}-\mu_{2} P_{05} P_{17} P_{5,14}+\mu_{1} P_{05} P_{29} P_{5,14} \\
& +\mu_{2} P_{05} P_{52}-\mu_{2} P_{05} P_{17} P_{52}+\mu_{1} P_{05} P_{29} P_{52} \\
& +\mu_{1} P_{06} P_{6,17}+\mu_{2} P_{06} P_{18} P_{6,17}-\mu_{1} P_{06} P_{2,10} P_{6,17} \\
& +\mu_{2} P_{06} P_{6,18}-\mu_{2} P_{06} P_{17} P_{6,18}+\mu_{1} P_{06} P_{29} P_{6,18} \\
& +\mu_{3} P_{06} P_{36}-\mu_{3} P_{06} P_{17} P_{36}-\mu_{3} P_{06} P_{2,10} P_{36} \\
& +\mu_{3} P_{06} P_{2,10} P_{17} P_{36}-\mu_{3} P_{06} P_{29} P_{18} P_{36} \\
& +\mu_{1} P_{06} P_{29} P_{3,15} P_{36}+\mu_{2} P_{06} P_{18} P_{3,15} P_{36} \\
& -\mu_{1} P_{06} P_{2,10} P_{3,15} P_{36}+\mu_{2} P_{06} P_{3,16} P_{36} \\
& -\mu_{2} P_{06} P_{17} P_{3,16} P_{36}+\mu_{1} P_{06} P_{29} P_{3,16} P_{36} \text {, }
\end{aligned}
$$

and

$$
\begin{aligned}
D_{0}= & -\mu_{0}\left(-P_{10}-P_{18} P_{20}+P_{10} P_{2,10}\right)-\mu_{1}\left(-P_{01}+P_{01} P_{2,10}\right. \\
& -P_{02} P_{29}-P_{03} P_{3,15}+P_{03} P_{2,10} P_{3,15}-P_{03} P_{29} P_{3,16} \\
& -P_{04} P_{41}+P_{04} P_{2,10} P_{41}-P_{04} P_{4,11}+P_{04} P_{2,10} P_{4,11} \\
& -P_{04} P_{2,29} P_{4,12}-P_{05} P_{5,13}+P_{05} P_{2,10} P_{5,13}-P_{05} P_{29} P_{5,14} \\
& -P_{05} P_{29} P_{52}-P_{06} P_{6,19}+P_{06} P_{2,10} P_{6,19}-P_{06} P_{29} P_{6,20} \\
& \left.-P_{06} P_{3,15} P_{63}+P_{06} P_{2,10} P_{3,15} P_{63}-P_{06} P_{29} P_{3,16} P_{63}\right) \\
& -\mu_{2}\left(-P_{02} P_{10}-P_{18}+P_{03} P_{18} P_{30}-P_{03} P_{10} P_{3,16}\right. \\
& -P_{04} P_{10} P_{4,12}-P_{05} P_{10} P_{5,14}-P_{05} P_{10} P_{52} \\
& \left.-P_{06} P_{10} P_{6,20}+P_{06} P_{18} P_{30} P_{63}-P_{06} P_{10} P_{63} P_{3,16}\right) \\
& -\mu_{3}\left(-P_{03} P_{10}-P_{03} P_{18} P_{20}+P_{03} P_{10} P_{2,10}\right. \\
& \left.-P_{06} P_{10} P_{63}-P_{06} P_{18} P_{63} P_{20}+P_{06} P_{10} P_{63} P_{2,10}\right) \\
& -\mu_{4}\left(-P_{04} P_{10}-P_{04} P_{18} P_{20}+P_{04} P_{15} P_{2,10}\right) \\
& -\mu_{5}\left(-P_{05} P_{20}+P_{05} P_{17} P_{20}-P_{05} P_{10} P_{29}\right) \\
& -\mu_{6}\left(-P_{06} P_{10}-P_{06} P_{18} P_{20}+P_{04} P_{10} P_{2,10}\right) .
\end{aligned}
$$

\section{BUSY PERIOD ANALYSIS}

\subsection{Expected busy period with repair due to hardware failure}

Upon using the probabilistic arguments, we obtain the following equations;

$$
B_{0}^{1}(t)=\sum_{i=1}^{i=6} q_{0 i}(t)\left(\mathcal{C} B_{i}^{1}(t),\right.
$$

$B_{1}^{1}(t)=\bar{G}_{1}(t)+q_{10}(t)\left(B_{0}^{1}(t)+q_{11}^{(7)}(t)\left(B_{1}^{1}(t)+q_{12}^{(8)}(t)\left(\mathcal{C} B_{2}^{1}(t)\right.\right.\right.$,

$B_{2}^{1}(t)=q_{20}(t) \subset B_{0}^{1}(t)+q_{21}^{(9)}(t) \subset B_{1}^{1}(t)+q_{22}^{(10)}(t)(c) B_{2}^{1}(t)$, 
$B_{3}^{1}(t)=q_{30}(t)\left(B_{0}^{1}(t)+q_{31}^{(15)}(t)\left(\mathcal{C} B_{1}^{1}(t)+q_{32}^{(16)}(t)\left(B_{2}^{1}(t)\right.\right.\right.$,

$$
B_{4}^{1}(t)=\left[q_{41}(t)+q_{41}^{(11,17)}(t)\right]\left(\mathcal{C} B_{1}^{1}(t)+q_{42}^{(12,8)}(t) \subset B_{2}^{1}(t),\right.
$$

$$
B_{5}^{1}(t)=q_{51}(t)\left(\mathcal{C} B_{1}^{1}(t)+\left[q_{52}(t)+q_{52}^{(14,10)}(t)\right]\left(\mathcal{C} B_{2}^{1}(t),\right.\right.
$$

$B_{6}^{1}(t)=q_{61}^{(17,15)}(t)\left(B_{1}^{1}(t)+q_{62}^{(18,16)}(t) \subset B_{2}^{1}(t)+q_{63}(t)\left(\mathcal{C} B_{3}^{1}(t)\right.\right.$.

Taking Laplace transform of Equations [(21)-(27)] and solving for $B_{0}^{1 *}(s)$, we get

$$
B_{0}^{1 *}(u)=\frac{N_{1}(u)}{D_{0}(u)} .
$$

The steady-state of the total fraction of time for which serverman is busy with hardware repair is given by

$$
B_{0}^{1}=\lim _{t \rightarrow \infty} B_{0}^{1}(t)=\lim _{u \rightarrow 0} u B_{0}^{1 *}(u)=\frac{N_{1}}{D_{0}},
$$

where

$$
\begin{aligned}
N_{1}= & \frac{1}{\nu_{1}}\left[P_{01}-P_{01} P_{29}+P_{03} P_{3,15}-P_{03} P_{2,10} P_{3,15}\right. \\
& +P_{03} P_{29} P_{3,16}+P_{04} P_{41}-P_{04} P_{2,10} P_{41} \\
& +P_{04} P_{4,11}-P_{04} P_{4,11} P_{2,10}+P_{04} P_{4,10} P_{29} \\
& +P_{05} P_{5,13}-P_{05} P_{2,10} P_{5,13}+P_{05} P_{29} P_{5,14} \\
& +P_{05} P_{29} P_{52}+P_{06} P_{6,17}-P_{06} P_{2,10} P_{6,17} \\
& +P_{06} P_{29} P_{6,18}+P_{06} P_{63} P_{3,15} \\
& \left.-P_{06} P_{2,10} P_{63} P_{3,15}+P_{06} P_{29} P_{3,16} P_{63}\right] .
\end{aligned}
$$

\subsection{Expected busy period with repair due to human failure}

Let $B_{k}^{2}(t)$ be the probability that the server man is busy with repair due to human failure at time $t$ starting form state $s_{i} \in E$. Upon using the probabilistic arguments, we obtain the following equations;

$$
B_{0}^{2}(t)=\sum_{i=1}^{i=6} q_{0 i}(t) \Subset B_{i}^{2}(t),
$$

$$
B_{1}^{2}(t)=q_{10}(t)\left(\mathcal{C} B_{0}^{2}(t)+q_{11}^{(7)}(t)\left(\mathcal{C} B_{1}^{2}(t)+q_{12}^{(8)}(t)\left(\mathcal{C} B_{2}^{2}(t),\right.\right.\right.
$$

$$
B_{2}^{2}(t)=q_{20}(t)\left(B_{0}^{2}(t)+q_{21}^{(9)}(t)\left(\mathcal{C} B_{1}^{2}(t)+q_{22}^{(10)}(t)\left(\mathcal{C} B_{2}^{2}(t),\right.\right.\right.
$$

$$
B_{3}^{2}(t)=\bar{V}(t)+q_{30}(t) \subseteq B_{0}^{2}(t)+q_{31}^{(15)}(t) \Subset B_{1}^{2}(t)+q_{32}^{(16)}(t) \subseteq B_{2}^{2}(t),
$$

$$
\begin{aligned}
& B_{4}^{2}(t)=\left[q_{41}(t)+q_{41}^{(11,17)}(t)\right] \text { ( } B_{1}^{2}(t)+q_{42}^{(12,8)}(t) \subset B_{2}^{2}(t) \\
& B_{5}^{2}(t)=q_{51}^{19,9}(t) \subseteq B_{1}^{2}(t)+\left[q_{52}(t)+q_{52}^{(14,10)}(t)\right] \subset B_{2}^{2}(t),
\end{aligned}
$$

$$
B_{6}^{2}(t)=q_{61}^{(17,15)}(t)\left(B_{1}^{2}(t)+q_{62}^{(18,16)}(t) \subseteq B_{2}^{2}(t)+q_{63}(t)\left(B_{3}^{2}(t) .\right.\right.
$$

Once again takin Laplace transforms of Equations [(30)- (36)] and solving for $B_{0}^{2 *}(s)$, we get

$$
B_{0}^{2 *}(u)=\frac{N_{2}(u)}{D_{0}(u)},
$$

The steady-state of expected busy period of the human error repair is given by

$$
B_{0}^{2}=\lim _{t \rightarrow \infty} B_{0}^{2}(t)=\lim _{u \rightarrow 0} u B_{0}^{2 *}(u)=\frac{N_{1}}{D_{0}} .
$$

Upon using Equations (38), we get

$$
\begin{aligned}
N_{2}= & \frac{1}{\nu_{2}}\left[P_{02}-P_{02} P_{17}+P_{01} P_{18}+P_{03} P_{18} P_{3,15}\right. \\
& +P_{03} P_{3,16}-P_{03} P_{17} P_{3,16}+P_{04} P_{18} P_{41} \\
& +P_{04} P_{18} P_{4,11}+P_{04} P_{4,12}-P_{04} P_{4,12} P_{17} \\
& +P_{05} P_{18} P_{5,13}+P_{05} P_{5,14}-P_{05} P_{5,14} P_{17} \\
& +P_{05} P_{52}-P_{05} P_{52} P_{17}+P_{06} P_{18} P_{6,17}+P_{06} P_{6,18} \\
& -P_{06} P_{17} P_{6,18}+P_{06} P_{18} P_{3,15} P_{63}+P_{06} P_{3,16} P_{63} \\
& \left.-P_{06} P_{17} P_{3,16} P_{63}\right] .
\end{aligned}
$$

\subsection{Expected Busy period with Standby Repair}

Let $B_{i}^{3}(t)$ be the probability that the server man is busy with the standby repair at time $t$ starting from state $S_{i} \in E$.

Using the probabilistic arguments, yields

$$
B_{0}^{3}(t)=\sum_{i=1}^{6} q_{01}(t)\left(B_{i}^{3}(t)\right.
$$

$$
\begin{aligned}
& B_{1}^{3}(t)=q_{10}(t)\left(B_{0}^{3}(t)+q_{11}^{(7)}(t) \subset B_{1}^{3}(t)+q_{12}^{(8)}(t)(C) B_{2}^{3}(t)\right. \\
& B_{2}^{3}(t)=q_{20}(t)\left(\mathcal{C} B_{0}^{3}(t)+q_{21}^{(9)}(t)\left(\mathcal{C} B_{1}^{3}(t)+q_{22}^{(10)}(t)\left(\mathcal{C} B_{2}^{3}(t)\right.\right.\right. \\
& B_{3}^{3}(t)=q_{30}(t)\left(B_{0}^{3}(t)+q_{31}^{(15)}(t) \subset B_{1}^{3}(t)+q_{32}^{(16)}(t) \subseteq B_{2}^{3}(t)\right. \text {, } \\
& B_{4}^{3}(t)=\left[q_{41}(t)+q_{41}^{(11,17)}(t)\right] \subset B_{1}^{3}(t)+q_{42}^{(12,8)}(t) \subset B_{2}^{3}(t) \\
& B_{5}^{3}(t)=q_{51}^{(19,9)}(t) \subset B_{1}^{3}(t)+\left[q_{52}(t)+q_{52}^{(14,10)}(t)\right] \subset B_{2}^{3}
\end{aligned}
$$

$B_{6}^{3}(t)=q_{61}^{(17,15)}(t)\left(\mathcal{C} B_{1}^{3}+q_{62}^{(18,16)}(t) \subseteq B_{2}^{3}(t)+q_{63}(t)\left(B_{3}^{3}(t)\right.\right.$. After some calculations, the steady-state expected busy period with standby repair can be given as follows

$$
B_{0}^{3}=\lim _{t \rightarrow \infty} B_{0}^{3}(t)=\lim _{u \rightarrow 0} u B_{1}^{3 *}(u)=\frac{N_{3}}{D_{0}},
$$

where $B_{1}^{3 *}(S)$ is LT of $B_{0}^{3}(t)$, and

$$
\begin{gathered}
N_{3}=\frac{1}{\eta}\left[P_{03}\left(1-P_{17}-P_{2,10}+P_{P_{1} 7} P_{2,10}-P_{18} P_{29}-P_{06} P_{17}\right)\right. \\
\left.-P_{06} P_{63}\left(P_{2,10}-P_{2,10} P_{17}+P_{29} P_{18}\right)\right] .
\end{gathered}
$$

\section{COST BENEFIT ANALYSIS}

Let $C(t)$ be the net revenue of the system in $(0, t]$, then

$$
C(t)=\alpha \mu_{u p}(t)-\sum_{i=1}^{i=3} \beta_{i} \mu_{R}^{i}(t),
$$

where

$\alpha$ is the revenue per unit of up time

$\beta_{i} ; \quad i=1,2,3$ are the cost per unit time of repair of the unit and the switch respectively.

$\mu_{u p}=\int_{0}^{t} A V_{0}(t) d t$

$\mu_{R}^{i}(t)=\int_{0}^{t} B_{0}^{i}(t) d t$ 
Taking LT of (40), gives

$$
C^{*}(u)=\alpha \mu_{u p}^{*}(u)-\sum_{i=1}^{3} \beta_{i} \mu_{R}^{i *}(u) .
$$

Define $C$ to represent the expected profit per unit of time in steady state.

So $C$ can be written as follows

$$
\begin{aligned}
C=C(\infty) & =\lim _{t \rightarrow \infty} \frac{C(t)}{t}=\lim _{u \rightarrow 0} u^{2} C^{*}(u) \\
& =\frac{\alpha N_{0}-\sum_{i=1}^{3} \beta_{i} N_{i}}{D_{0}} .
\end{aligned}
$$

\section{NUMERICAL EXAMPLE}

Setting $\alpha=300, \beta_{1}=20, \beta_{2}=10$ and $\beta_{3}=5, n=2$, $\lambda_{2}=0.002, P=0.8, q=0.2, \nu_{1}=0.08, \nu_{2}=0.02, \eta=0.06$ and $\xi=0.04$.

Figures (1-4) represent the variation of MTTF, $A V_{0}, B_{0}^{2}, B_{0}^{3}$ and $C$ versus $\lambda_{1}$ when $\nu_{1}<\nu_{2}, \nu_{1}=\nu_{2}$ and $\nu_{1}>\nu_{2}$.

Note: To save the space, figures versus $\lambda_{2}$ are omitted because they have the same manner.

REMARK 2. From Figures (1,2 and 4), we can see that

1- The MTTF, $A V_{0}$ and $C$ decreases as $\lambda_{1}$ increases

2- From Fig (3) the expected Basy period $B_{1}^{0}, B_{2}^{0}$ and $B_{3}^{0}$ increase as $\lambda_{1}$ increases.

\section{SPECIAL CASES}

1- Setting $n=1$, we get the results for two-unit warm standby based on imperfect repair facility and two types of failures.

2- Setting $n=1$ and $\gamma=0$, the results for two-unit cold standby system based on imperfect repair facility and two types failures.

3- Setting $n=1, \gamma=0$ and $P=1$, we obtain the result for two-unit cold standby system based on two types of failure.

\section{CONCLUSION}

The stochastic behaviour of $(n+1)$-unit warm standby system based on imperfect repair facility and two types of failure are studied. Some measures of reliability for the system are derived in the steady state. Based on a numerical example, it has been showed that MTTF, $A V_{0}$ and $C$ decrease as $\lambda_{1}$ or $\lambda_{2}$ increases while $B_{0}^{1}, B_{0}^{2}$ and $B_{0}^{3}$ are increasing as $\lambda_{1}$ or $\lambda_{2}$ increases.

\section{Acknowledgements}

The authors would like to thank Deanship of Scientific Research, King Abdul Aziz University for supporting this research project number (3-031/429).

\section{REFERENCES}

[1] R. K. Agnihotri and S. K. Statsangi, Analysis of a two-unit redundant system with optimum interchangement time, Reliab. 35 (1995), no. 4, 749-750.

[2] B. S. Dhilon, Om human reliability bibliography, Microelectron. Reliab. 20 (1980), 371-373.

[3] B. S. Dhilon, Stochastic models for predicting human reliability, Microelectron. Reliab. 22 (1982), 491-496.

[4] B. S. Dhilon and R. B. Mishra, Reliability evaluation of system with critical human error, Microelectron. Reliab. 24 (1984), 743-759.

[5] B. S. Dhilon and S. N. Rayayoti, Reliability analysis of non-maintained parallel systems subject to hardware failure and human error, Microelectron. Reliab. 25 (1985), $111-122$
[6] P. P. Gupta and Arvind Kumar, Reliability and mttf analysis of a non-repairable parallel redundant complex system under hardware and human failures, Microelectron. Reliab. 29 (1986), 229-234.

[7] P. P. Gupta and R. K. Sharma, Reliability analysis of a twostate repairable parallel redundant system under human failure, Microelectron. Reliab. 29 (1986), 221-224.

[8] E. W. Hogen, Human reliability analysis, Nuclear Safety 17 (1976), 315-326.

[9] S. k. Singh, R. P. Singh, and S. Sindhu, Cost-benefit analysis of 2-unit priority standby system with patience-time for repair, IEEE Trans. Reliab. 40 (1991), no. 1, 11-14.

[10] J. C. Ke and Y. K. Chu, Comparative analysis of availability for redundant repairable system, Appl. Math. Comput. 188 (2007), 332-338.

[11] A. Kumar, S. K. Gupta, and G. Taneja, Probabilistic analysis of a two-unit cold standby system with instructions at need, Microelectron. Reliab. 35 (1995), no. 5, 829-832.

[12] X. Li, R. Yan, and M. Xuo, Evaluating a warm standby system with components having proportional hazard rates, Oper. Res. Lett. 37 (2009), 56-60.

[13] M. A. W. Mahmoud and M. M. Al Ameel, Stochastic behavior of a two-unit cold standby redundant system with imperfect repair facility subject to hardware and human error failures, Proceeding of MS'2002 International Conference on Modeling and Simulation in Technical and Social Sciences (Gerona, Catalonia, Spain), June 2002, pp. 25-27.

[14] M. A. W. Mahmoud, Mohei El-Din, and M. El-Said Moshref, Optimum preventive maintenance for a two-unit priority standby system with patienc-time for repair, Optimization 29 (1994), 361-379.

[15] M. A. W. Mahmoud and M. A. Esmail, Stochastic analysis of two-unit warm standby system with switch subject to hardware and human error failures, Microelectron. Reliab. 38 (1998), 1639-1644.

[16] M. A. W. Mahmoud and M. El-Said Moshref, On a 2-unit cold standby system considering hardware, human error failures and preventive maintenance, Math. Comp. Model. (2010).

[17] D. Meister, The problem of human initiated failures, $8^{\text {th }}$ National Symposium on Reliability and Quality Control, 1962.

[18] K. Wang and L. Chiu, Cost benefit analysis of availability systems with warm standby unit and imperfect coverage, Appl. Math. Comput. 172 (2006), 1239-1256. 


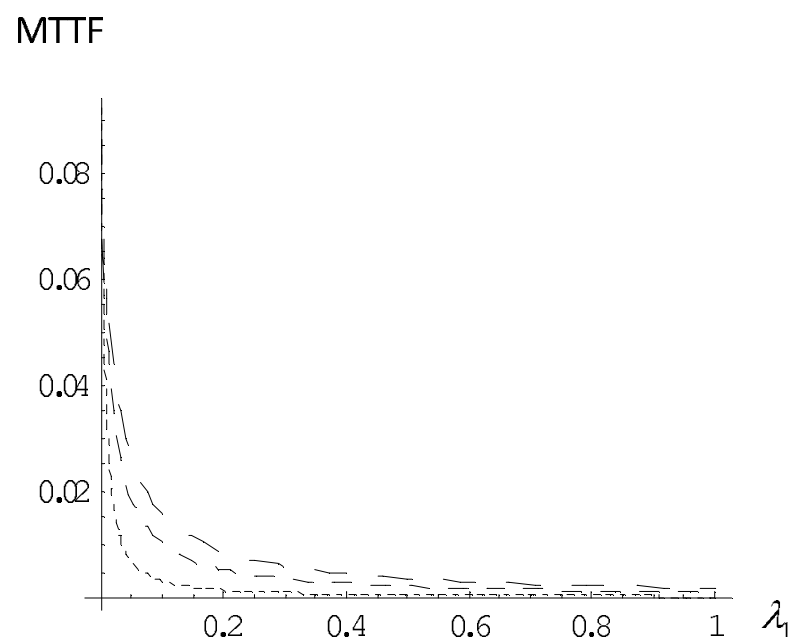

Fig (1): Variation of MTTF versus $\lambda_{1}$

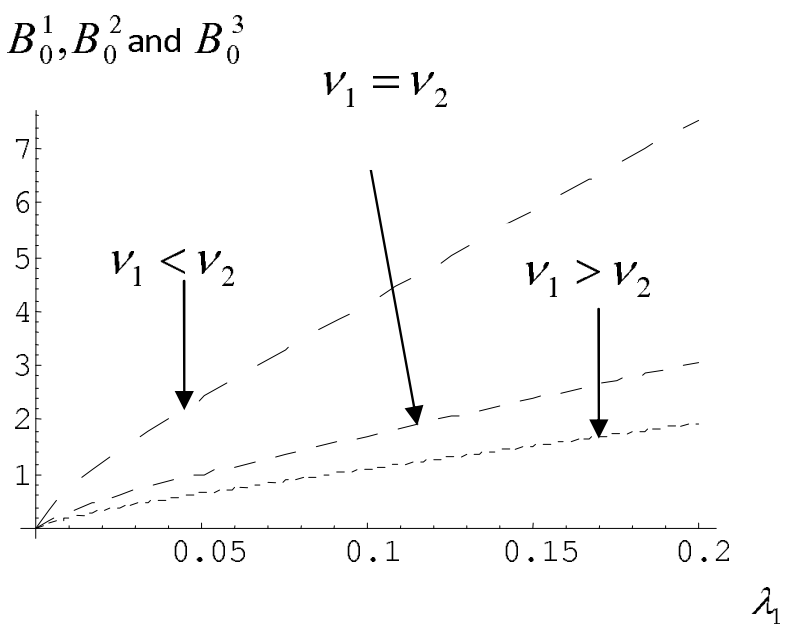

Fig (3): Variation of $B_{0}^{1}, B_{0}^{2}$ and $B_{0}^{3}$ versus $\lambda_{1}$
$A v_{0}$

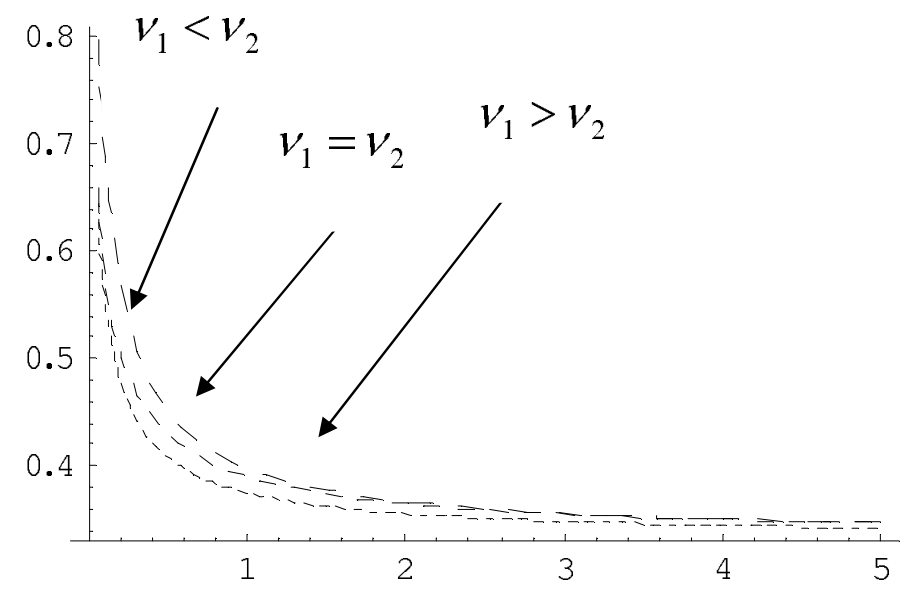

$\lambda_{1}$

Fig (2): Variation of $A V_{0}$ versus $\lambda_{1}$

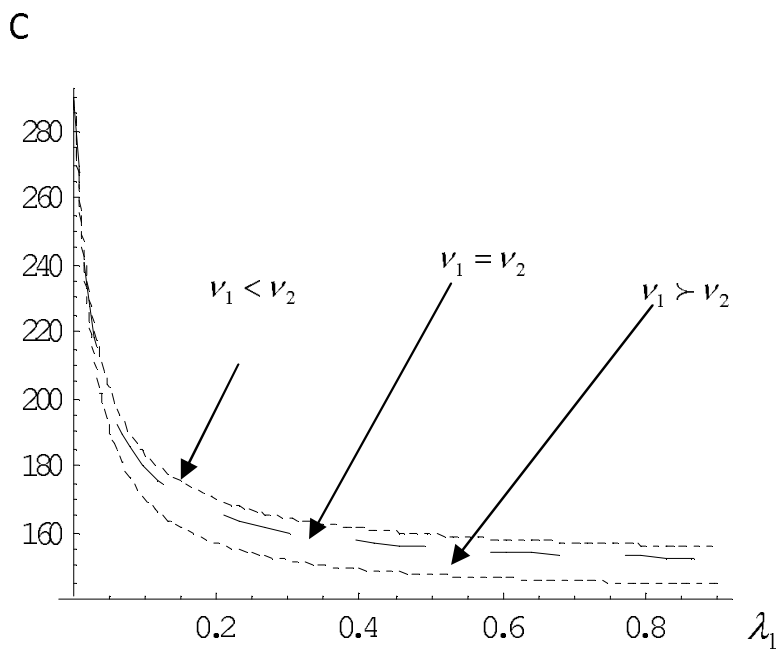

Fig (4): Variation of $C$ versus $\lambda_{1}$ 